\title{
A practical device for pinpoint delivery of molecules into multiple neurons in culture
}

\author{
Chikako Hara ${ }^{1,2}$, Kiyohiko Tateyama ${ }^{3}$, Naoki Akamatsu ${ }^{3}$, Hiroyuki Imabayashi ${ }^{3}$, Koichi Karaki $^{3}$, Nobuo \\ Nomura $^{4}$, Hideyuki Okano ${ }^{2}$ and Atsushi Miyawaki ${ }^{1, *}$ \\ ${ }^{1}$ Laboratory for Cell Function Dynamics, Advanced Technology Development Group, Brain Science Institute, \\ RIKEN, 2-1 Hirosawa, Wako-city, Saitama 351-0198, Japan (*author for correspondence; e-mail: \\ matsushi@brain.riken.jp) \\ ${ }^{2}$ Department of Physiology, Keio University School of Medicine, 35 Shinanomachi, Shinjyuku-ku, Tokyo \\ 160-8582, Japan \\ ${ }^{3}$ MEMS Department, MEMS Technology Division, Olympus Corporation, 2-3 Kuboyama-cho, Hachioji-shi, \\ Tokyo 192-8512, Japan \\ ${ }^{4} J a p a n$ Biological Information Research Center, 2-41-6 Aomi, Koto-ku, Tokyo 135-0064, Japan
}

Received 10 November 2007; Revised 16 January 2008; Accepted 28 January 2008

Published online 5 April 2008

(c) Springer Science+Business Media, LLC 2008

We have developed a device for pinpoint delivery of chemicals, proteins, and nucleic acids into cultured cells. The principle underlying the technique is the flow of molecules from the culture medium into cells through a rupture in the plasma membrane made by a needle puncture. DNA transfection is achieved by stabbing the needle tip into the nucleus. The CellBee device can be attached to any inverted microscope, and molecular delivery can be coupled with conventional live cell imaging. Because the position of the needle relative to the targeted cultured cells is computer-controlled, efficient delivery of molecules such as rhodamine into as many as $100 \mathrm{HeLa}$ cells can be completed in $10 \mathrm{~min}$. Moreover, specific target cells within a single dish can be transfected with multiple DNA constructs by simple changes of culture medium containing different plasmids. In addition, the nanosized needle tip enables gentle molecular delivery, minimizing cell damage. This method permits DNA transfection into specific hippocampal neurons without disturbing neuronal circuitry established in culture.

\section{Introduction}

Several methods have been developed for the transfer of chemicals, proteins, and nucleic acids into live cells (Stephens and Pepperkok, 2001). Most conventional methods, including carrier-mediated transfer (Felgner et al., 1987) and electroporation, aim to transfect an entire cell population, while target cell transfection has typically been achieved using glass pipettes to microinject (Graessmann et al., 1974). Recently, new methods of transfecting individual cells have been developed with the help of advanced technologies, such as femtosecond lasers (Stevenson et al., 2006), atomic force microscopy (AFM) probes (Cuerrier et al., 2007), and carbon nanotubes (Chen et al., 2007; Han et al., 2005). Transfection of individual live cultured cells at high spatial resolution has been achieved using AFM with an ultrathin needle sharpened to a diameter of 200-300 nm by focused ion beam etching (Tseng, 2005). The AFM tip was decorated with DNA encoding green fluorescent protein (GFP) and was inserted into cells and held there for some time, resulting in accumulation of 
Hara et al.

Fig. 1. The CellBee device attached to an inverted confocal microscope. (A) A diagram of a laser confocal microscopy system (FV300, Olympus) with an inverted microscope (IX81, Olympus), onto which the CellBee device is attached. SU, scanning unit; PMT, photomultiplier tube; PC, personal computer. (B) A schematic of the fine needle. (C) A view of the CellBee device attached to the IX81. (D) An expanded view corresponding to the dotted box in B. (D) An expanded view corresponding to the dotted box in $\mathrm{C}$.

GFP fluorescence in the treated cells (Obataya et al., 2005). Repeated insertion of DNA-decorated AFM probes into cultured cells resulted in a transfection rate of $30 \%$ (Cuerrier et al., 2007).

Here, we describe a simple, efficient, and gentle method for molecular delivery into cultured cells. We have developed a device with an ultrathin needle that can be attached to any inverted microscope. To simplify the procedure, interactions between the tip end and the cell membrane are not monitored, and molecules for delivery are added to the culture medium. Using a three-dimensional stage controller, as many as 100 cells can be transfected within $10 \mathrm{~min}$. DNA and chemical (Rhodamine101) delivery into HeLa cells was achieved with high success rates $(75 \%$ and $100 \%$, respectively), and DNA was transfected into cultured neurons without causing any damage.

\section{Results}

To improve the means of molecular delivery into individual cells, we developed an apparatus called a CellBee that can be attached to an inverted microscope (IX81, Olympus) (Fig. 1A). A silicon needle composed of a tip and a lever is attached to a motor-controlled z-stage (LAS20-025-52S, SMAC JAPAN) via an anchor and a holder (Fig. 1 $B)$. This apparatus is attached to the condenser component of the inverted microscope so that the tip is oriented vertically (Fig. 1C, D). The needle can be mobilized along the $z$-axis with a resolution of $0.1 \mu \mathrm{m}$. Cells cultured on a coverslip are set on a stage, and the medium is replaced with Hank's solution supplemented with the molecules to be delivered. Cell morphology is monitored by phase contrast $(\mathrm{PC})$ or differential interference contrast (DIC). The 488-nm beam from an $\mathrm{Ar}^{+}$laser is introduced through the objective, and the reflected
A practical device for pinpoint delivery of molecules

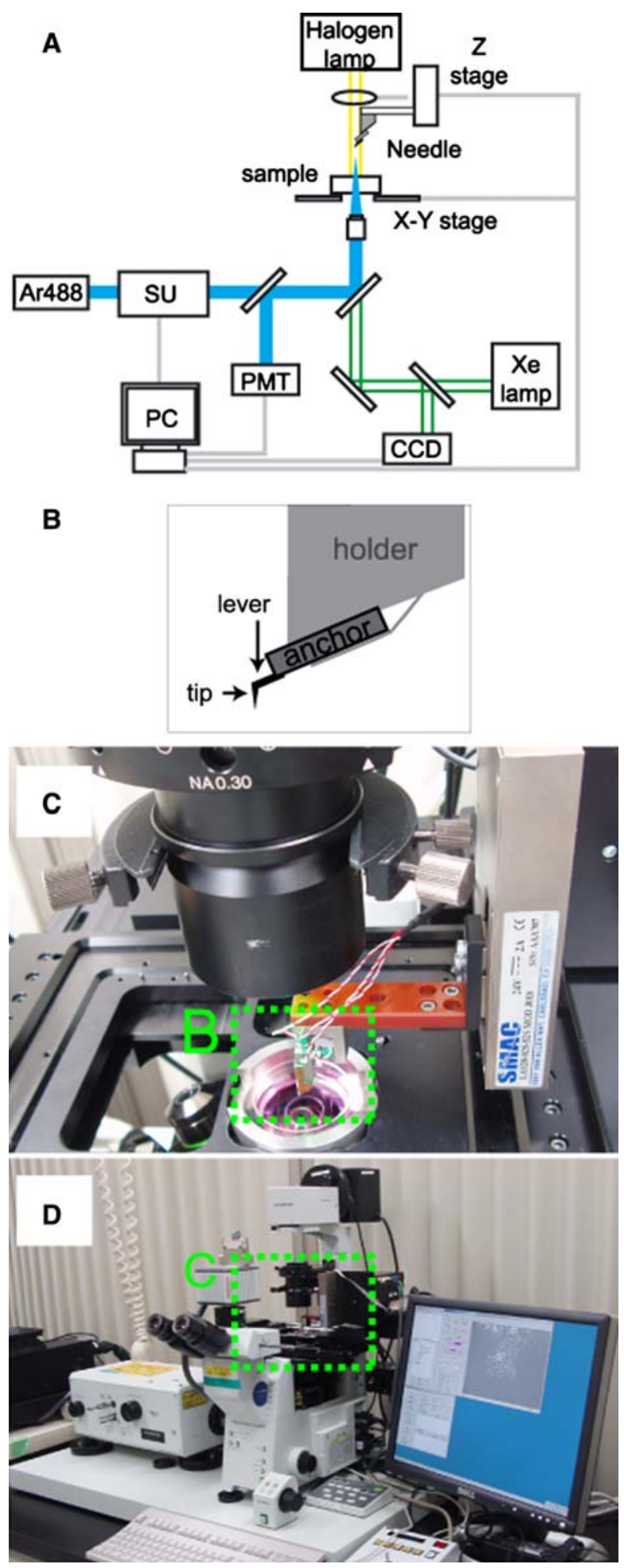


Hara et al.
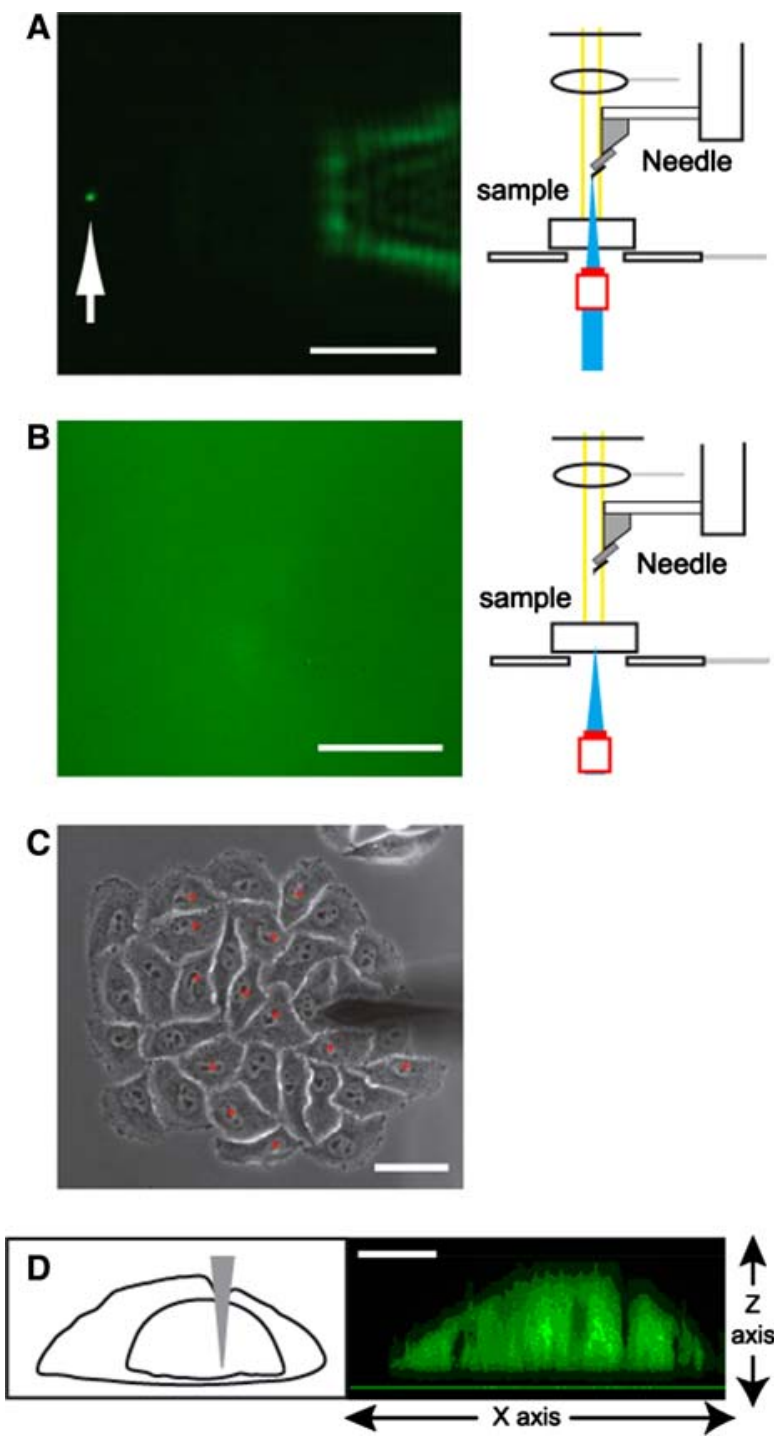

Fig. 2. Execution of cell stabbing using the CellBee. (A, B) The scattering images (left) and focus positions (right) of the laser beam $(488 \mathrm{~nm})$ for detecting the needle tip $(A)$ and the bottom of a coverslip (B). (C) A PC image of the needle over a colony of HeLa cells. Scale bars in (A), (B), and (C) are $50 \mu \mathrm{m}$. (D) A side view when the needle tip was stabbed into the nucleus of a HeLa cell. Scale bar, $5 \mu \mathrm{m}$.

light that passes through a pinhole is detected using a photomultiplier tube (PMT). This laserscanning confocal microscopy can determine the $z$-position of the tip end (Fig. 2A) and the bottom of the coverslip (Fig. 2B).

Delivery of molecules is carried out by positioning the tip over a cell of choice (Fig. 2C), and then lowering the needle until the tip end reaches a
A practical device for pinpoint delivery of molecules

fixed distance above the upper surface of the coverslip (Fig. 2D). This technique does not monitor interactions between the tip and cell membrane, whereas previously reported techniques using AFM probes measured force-distance curves (Cuerrier et al., 2007). Once the z-positions of the tip and coverslip are registered, it is possible to perform delivery in a programmed fashion. Therefore, the CellBee is best used in combination with an $x-y$ stage controller for pinpoint delivery to multiple cells with high accuracy and speed by selecting cells using a PC image and then automatically and sequentially injecting cells. Homemade software coordinates the image acquisition and stage control. The coverslip is placed on a motor-controlled $x-y$ stage (BIOS-212T, SIGMAK$\mathrm{OKI})$, and an image of the cells on the coverslip is
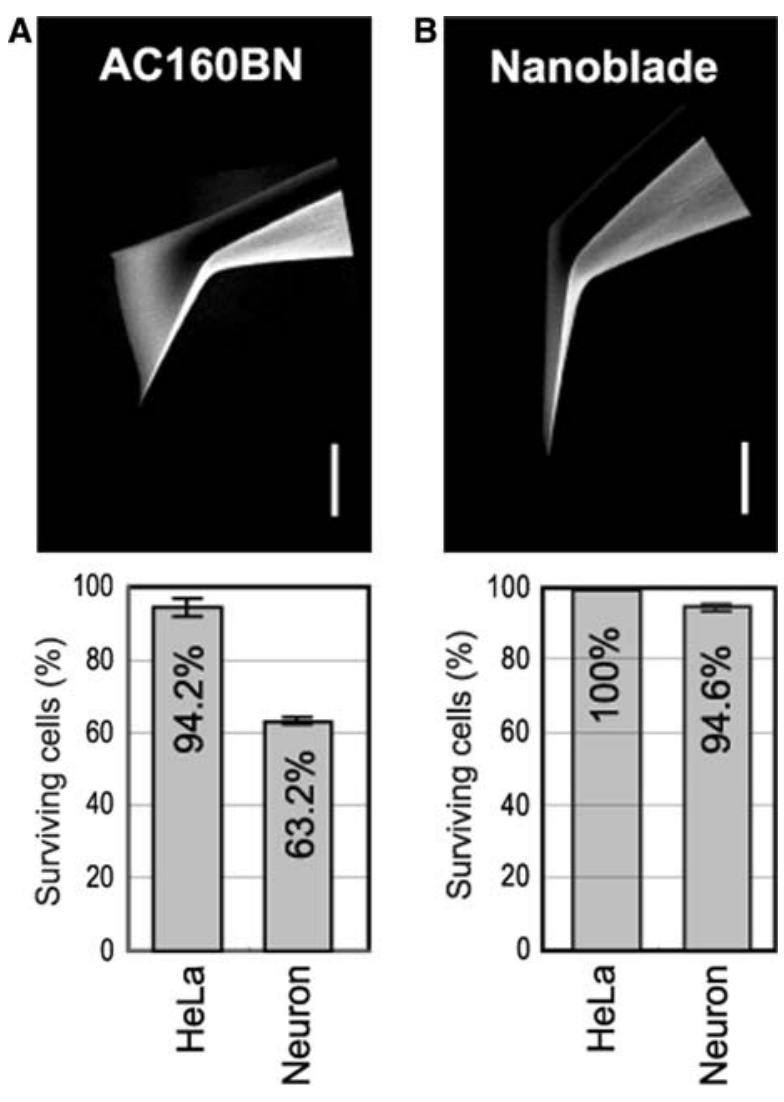

Fig. 3. Survival of cells stabbed with dull and fine needle tips. (Top) SEM images of the needle tips. Scale bar, $5 \mu \mathrm{m}$. (Bottom) Survival rates of HeLa cells and neurons stabbed with the needles (mean $\pm \mathrm{SE}, n=5$ for HeLa cells; $n=3$ for neurons). (A) AC160BN. (B) NanoBlade. 
Hara et al.
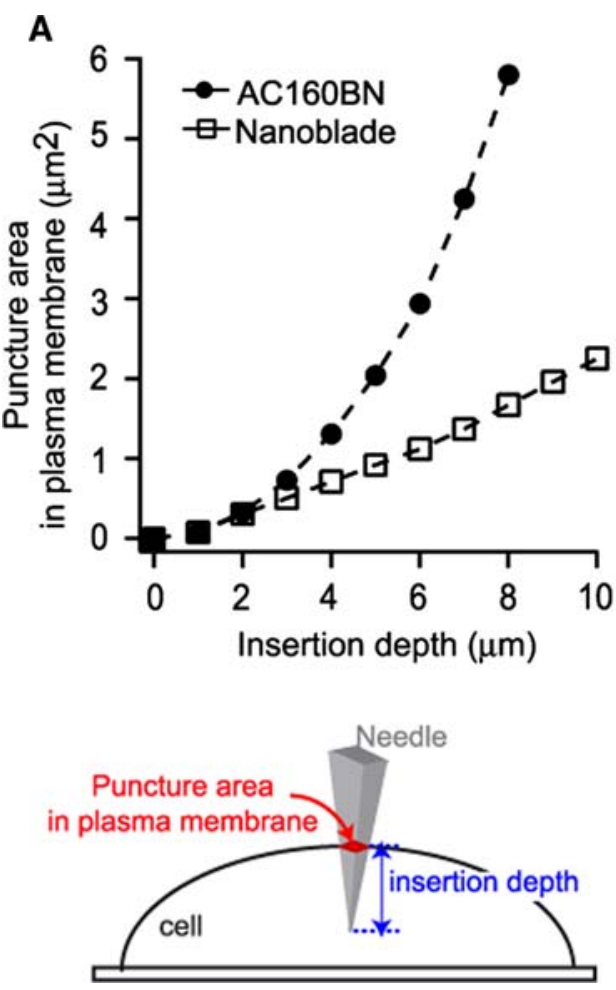

A practical device for pinpoint delivery of molecules

B
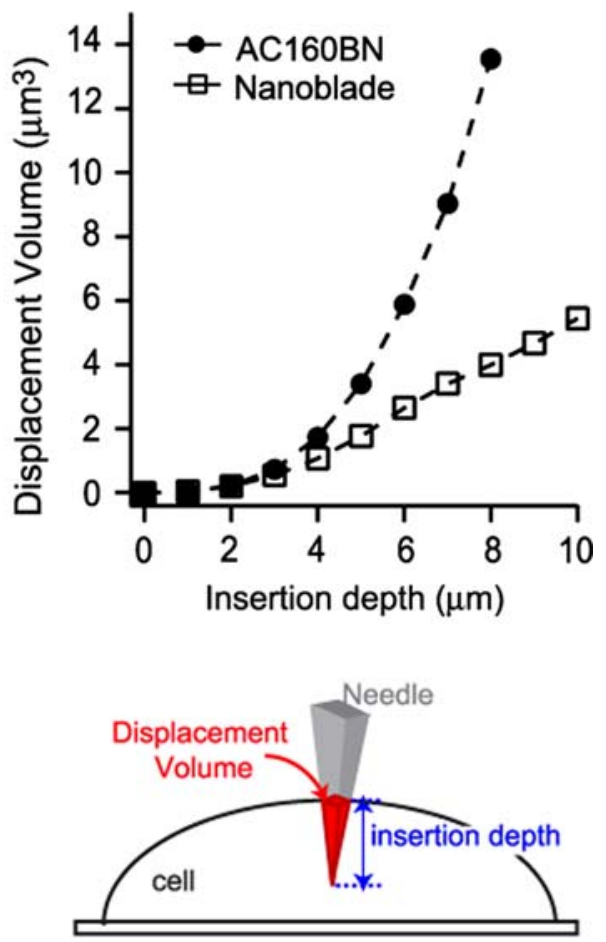

Fig. 4. Comparison of plasma membrane puncture area and displacement volume between a conventional AFM needle and the NanoBlade.

taken by a CCD camera (ORCA-ER, HAMAMATSU) and displayed on a screen. The position of each cell is defined by its $x-y$ coordinates.

To develop a suitable needle, conventional AFM cantilevers made of silicon were first tested. We noticed that the flexibility of the needle was critical, with a very flexible cantilever failing to penetrate cell membranes, and a rigid cantilever breaking easily when its tip touched the surface of the coverslip. Thus, we chose a cantilever with moderate flexibility (AC160BN, Olympus). As revealed by an SEM image (Fig. $3 A$ ), the tip of the AC160BN needle was not extremely fine. To avoid possible cell damage caused by insertion of such a dull tip, we shaved the AC160BN using focused ion beam (FIB) technology. The sharpened cantilever (Fig. 3B) was given the name "NanoBlade."
Assuming that the tips of AC160BN and the NanoBlade pierced into round cells, we calculated the area of the puncture of the plasma membrane (Fig. 4A) and the volume of the cell displaced with the tip (Fig. 4B) based on SEM images (Fig. 3). While both the area and volume increased with the insertion depth, an almost linear relationship was calculated for the NanoBlade, whereas a supralinear relationship was determined for the AC160BN needle. This suggests that the NanoBlade causes much less cell damage than an AC160BN needle.

Since the NanoBlade caused a small displacement volume, we were concerned about whether or not a fine needle could introduce a sufficient amount of chemical dye for identification of stabbed cells. Thus, we performed stabbing of HeLa cells in the presence of Rhodamine101 $(20 \mu \mathrm{g} / \mathrm{ml})$ in the

Table 1. Efficient, gentle delivery of chemicals or DNA into HeLa cells

\begin{tabular}{lclcc}
\hline & Stabbed cells & Dead cells & Fluorescent cells & Efficiency (\%) \\
\hline Rhodamine delivery & 105 & 0 & 105 & 100 \\
Expression of FL protein & 1715 & 0 & 1297 & 75 \\
\hline
\end{tabular}


Hara et al.
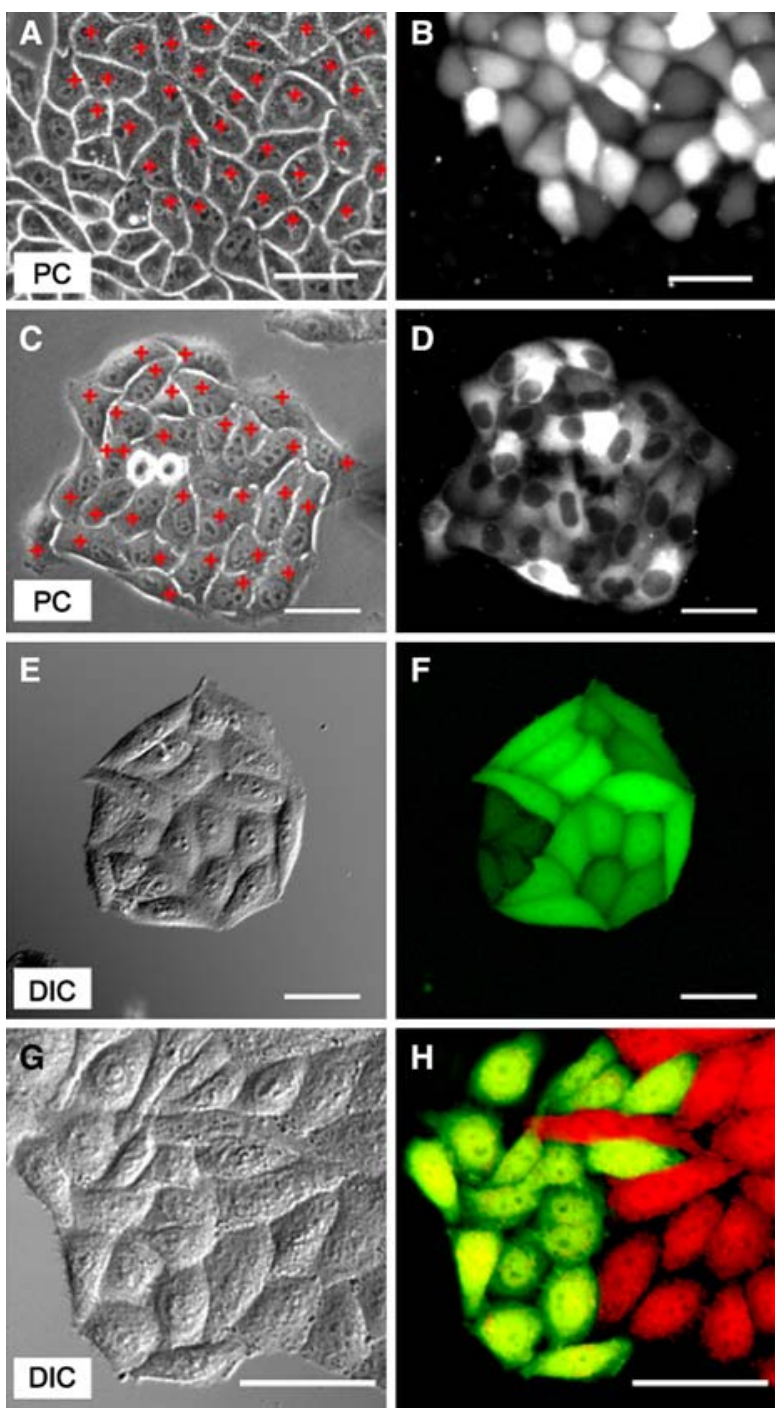

Fig. 5. Delivery of chemicals, proteins, or DNA into HeLa cells. (A, B) Delivery of Rhodamine101. A PC image of a confluent monolayer of HeLa cells. Stab points are indicated by red crosses (A). A fluorescence image acquired immediately after washing the medium (B). (C, D) Delivery of recombinant DsRed. A PC image of a colony of HeLa cells. Stabbed points (cytosol) are indicated by red crosses $(C)$. A fluorescence image acquired immediately after washing the medium (D). (E, F) A DIC image (E) and fluorescence image (F) of a colony of HeLa cells $24 \mathrm{~h}$ post-transfection with Venus plasmid DNA. (G, H) A DIC image (G) and fluorescence image $(H)$ of HeLa cells $24 \mathrm{~h}$ after differential transfection of Venus and mRFP1 plasmid DNA. Scale bar, $50 \mu \mathrm{m}$.

medium. One hundred and five cells were selected on a PC image (a part of the image is shown in Fig. 5A), and cells were stabbed sequentially with
A practical device for pinpoint delivery of molecules

the CellBee $z$-stage and the $x-y$ stage controllers fully active. The whole process took only $10 \mathrm{~min}$. Immediately after washing with normal medium, we observed that all of the stabbed cells showed a detectable amount of red fluorescence (Table 1), although the fluorescence intensity varied from cell to cell. A fluorescence image corresponding to the $P C$ image (Fig. $5 A$ ) is shown in Fig. $5 B$. All the stabbed cells survived for $24 \mathrm{~h}$ (results not shown), suggesting that the cells were not significantly damaged. Using a similar protocol, recombinant DsRed protein was also introduced into HeLa cells (Fig. 5C, D). In this experiment, selective introduction into the cytosol was attempted. The tetrameric complex of DsRed was indeed distributed in the cytosol and not the nucleus of treated HeLa cells.

Next, we tried DNA transfection into cultured HeLa cells using the CellBee system. The culture medium was supplemented with $0.1 \mathrm{mg} / \mathrm{ml}$ plasmid DNA (pVenus-N1) encoding Venus (Nagai et al., 2002), a bright version of yellow fluorescent protein. Cells were stabbed with the needle tip in their nuclei. To determine the percentage of cells transformed by pVenus-N1 DNA, cellular morphology and fluorescence were observed after $24 \mathrm{~h}$ by acquiring a DIC (Fig. 5E) and fluorescence image (Fig. 5F). Similar experiments were repeated 11 times. Taking into account the increase in the number of cells due to cell division over $24 \mathrm{~h}$, we determined that $75 \%$ of the stabbed cells had been transformed, and that no cells died from the cell surgery.

Differential transfection of targeted cells with two distinct plasmid DNA constructs is also possible. After some HeLa cells had been stabbed in the medium containing pVenus- $\mathrm{N} 1(0.1 \mathrm{mg} / \mathrm{ml})$, the medium was replaced with that containing mRFP1 cDNA (mRFP1/pcDNA3, $0.1 \mathrm{mg} / \mathrm{ml}$ ) and new cells were stabbed. After $24 \mathrm{~h}$, two populations of HeLa cells with yellow or red fluorescence emerged (Fig. 5G, H). It is notable that the boundary between the two cell populations was clear. This differential transfection technique may be useful for studying the function of membrane-bound heterophilic molecules involved in cell adhesion or repulsion.

In order to demonstrate that stabbing with the NanoBlade does not cause significant cell damage, we attempted the CellBee technique on more fragile primary cells. Transfection of cultured hippocampal neurons with mRFP1 cDNA was performed $(0.1 \mathrm{mg} / \mathrm{ml} \mathrm{mRFP} 1 / \mathrm{pcDNA} 3$ in the medium). Because primary neurons do not proliferate, it is relatively easy to identify stabbed 

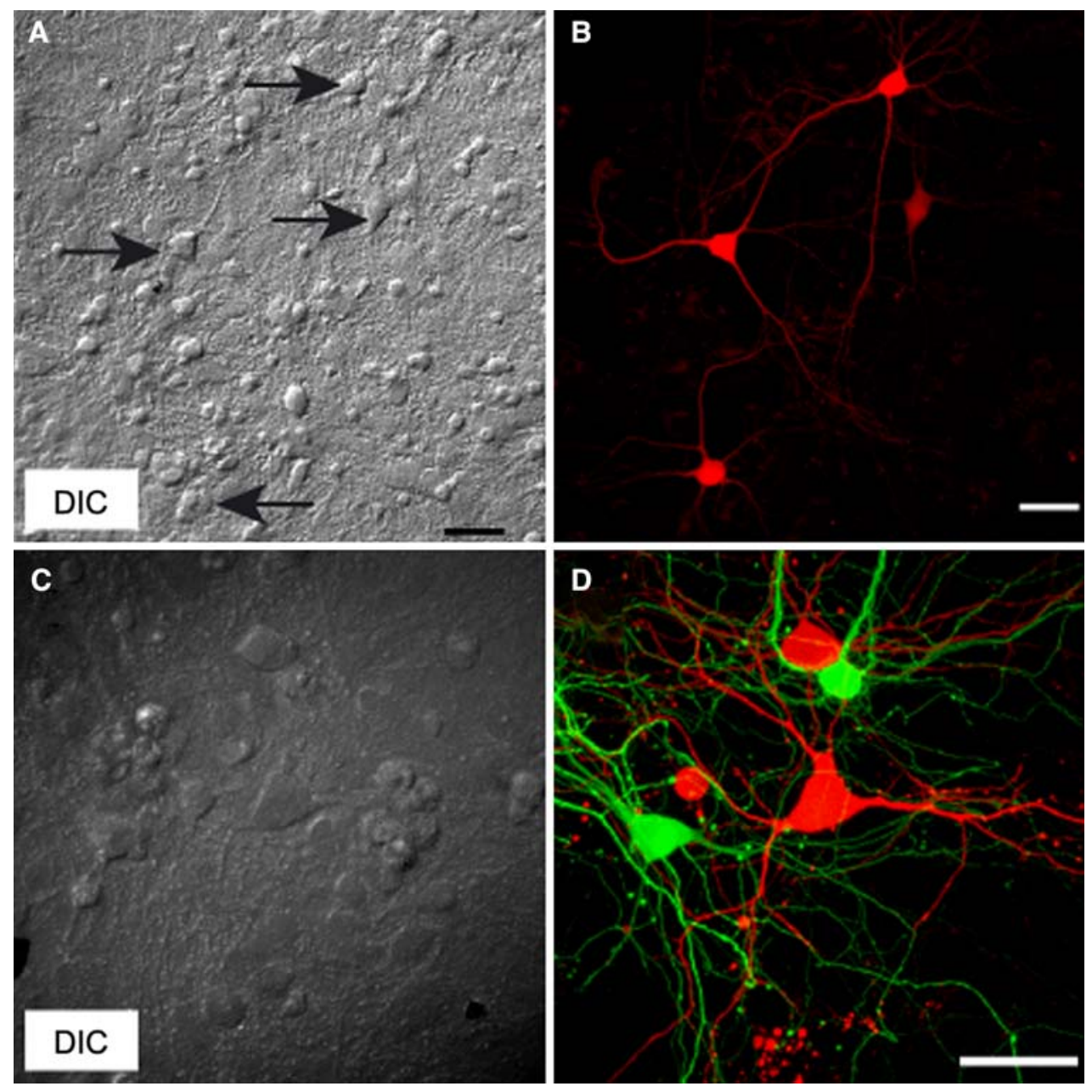

Fig. 6. DNA transfection of primary cultured neurons. (A, B) A DIC image (A) and a fluorescence image (B) of primary hippocampal neurons $24 \mathrm{~h}$ post-transfection with mRFP1 plasmid DNA. Stabbed neurons are indicated by arrows in A. (C, D) A DIC image (C) and fluorescence image (D) of primary hippocampal neurons $24 \mathrm{~h}$ after differential transfection of Venus and mRFP1 plasmid DNA. Scale bar, $50 \mu \mathrm{m}$.

neurons in images acquired $24 \mathrm{~h}$ post-transfection. Stabbed neurons are indicated by arrows in a DIC image (Fig. 6A). A fluorescence image shows that the same neurons emitted red fluorescence (Fig. 6 B). Furthermore, dual-color labeling of hippocampal neurons in a circuit is possible (Fig. 6C, D). This technique results in extremely high viability of stabbed neurons $(96 \%)$, compared with previous results using a conventional AFM cantilever $(64 \%)$.

\section{Discussion}

Pinpoint molecular delivery into cultured cells is generally performed using a glass pipette that approaches the cell at a $45^{\circ}$ angle (Graessmann et al., 1974). Such oblique needle positioning is disadvantageous for the following reasons: First, because the position of the pipette tip relative to a targeted cell is not regular, rapid delivery into numerous cells is hard to perform. Second, in the case of DNA transfection, in which a pipette tip must reach the nucleus, the Golgi apparatus next to the nucleus is often damaged. By contrast, the CellBee system described in this article uses a needle that is oriented and moved vertically. Thus, the $x-y$ position of the needle tip is always identifiable in PC or DIC images, and damage to the Golgi apparatus can be avoided. Furthermore, the CellBee technique does not monitor interactions 
Hara et al.

between the needle tip and the plasma membrane of a targeted cell, thus simplifying the process. Instead, the needle pierces the cell or nucleus at a pre-set distance above the upper surface of the coverslip. In our experiments, delivery of chemicals or DNA into about 100 cells was achieved in a short period of time ( $<15 \mathrm{~min}$ ) with high cell viability.

Previous studies demonstrated that single cell transfection was possible using AFM probes decorated with plasmid DNA (Cuerrier et al., 2007). Those studies extensively investigated the interaction between probes and cell membranes by measuring AFM force-distance curves, and their transfection techniques were performed on relatively robust cells, such as HEK293 cells. In contrast, we propose here a device that makes the AFM-based molecular delivery technique more practical for cell biologists, using conventional light microscopy systems.

Another rapidly expanding technique for the introduction of macromolecules into single cells is photoporation. Targeted transfection by intense near-infrared femtosecond laser pulses is particularly appealing to many biologists (Tirlapur and König, 2002), but photoporation requires much experience and expertise, and the equipment is fairly expensive and large. While electroporation usually transfects an entire cell population, target cell electroporation has also been successfully performed (Hass et al., 2001; Olofsson et al., 2003). However, this technique does not permit transfection of a number of cells in a short period of time, or selective delivery into the cytoplasm or nucleus. The CellBee method we describe here uses a mechanical means of delivery, thereby permitting high spatiotemporal control. It has the advantage of simple and easy manipulation, as well as gentle treatment of fragile cell types, such as cultured neurons.

Cultured neurons make synapses with one another to establish functional circuits on a coverslip. Co-cultured glial cells (astrocytes) also actively participate in the development and maintenance of neuronal circuitry (Stevens, 2003; Hama et al., 2004). To analyze the roles of adhesive/repulsive and signaling molecules in synaptogenesis or synaptic plasticity in the context of circuit formation, it is necessary to transfect specifically targeted neurons or astrocytes with different plasmid DNA constructs and examine the effects on cell-cell communication. Since cultured, post-mitotic neurons participating in a circuit
A practical device for pinpoint delivery of molecules

cannot easily be replaced when damaged, it is also important to preserve transfected neurons. Our CellBee device is able to achieve both of these objectives, using a simple change of medium to transfect different plasmids and a nano-sized needle tip that permits high viability of transfected neurons.

\section{Methods}

\section{HeLa cell preparation}

HeLa cells were grown on the glass-bottom dish in Dulbecco's modified Eagles medium (DMEM, Sigma) containing $10 \%$ FBS.

\section{Neuron preparation}

Primary hippocampal neurons were prepared from Wistar rat fetuses (embryonic day 18-20) and plated on a poly-L-Lysine coated glass-bottom dish in Minimum Essential Medium Eagle (MEM, Sigma) containing $2 \%$ FBS and N2 supplement.

\section{Plasmid DNA preparation}

pVenus-N1, mRFP1/pcDNA3, and EGFP/pcDNA3 were amplified in Escherichia coli and purified using the endo-toxin free kit (QIAGEN).

\section{DsRed protein preparation}

Recombinant DsRed protein fused to His-tag was produced in E. coli and purified using the Ni-NTA agarose (Mizuno et al., 2001).

\section{Scanning electron microscopy}

Scanning electron microscopy (SEM) images of needles were taken by Field-emission scanning electron microscopy FESEM (S-4700, Hitachi) in a 4000 times expand mode.

\section{Construction of the CellBee device}

The motor-controlled z-stage (LAS20-025-52S) was purchased from SMAC JAPAN (http:// www.smac-mca.co.jp/). AC160BN needles were etched with gallium ions using DualBeam SEM/ FIB (Nova 600 NanoLab) to make Nanoblades.

\section{Injection protocol into cells}

(1) The $x-y$ position of the needle was determined using a screw adjuster on the CellBee and was almost always placed in the center of the field 
of view. The $z$ position of the needle tip was adjusted by measuring reflected light.

(2) The culture medium was replaced with Hank's Balanced Salt Solution (HBSS, GIBCO) containing $0.1 \mu \mathrm{g} / \mu \mathrm{l}$ plasmid DNA, $20 \mu \mathrm{g} / \mathrm{ml}$ Rhodamine (Rhodamine101, DOJINDO), or $1 \mathrm{mg} /$ $\mathrm{ml}$ DsRed protein. The solution contained $1.3 \mathrm{mM} \mathrm{CaCl}_{2}$.

(3) A target cell was placed under the needle by the $x-y$ stage controller. The needle was lowered vertically to pierce the cell membrane. The needle tip stayed inside the cell for one second.

(4) Cells were washed with fresh culture medium a few times, followed by incubation in $\mathrm{a} \mathrm{CO}_{2}$ incubator.

Measurement of survival rates of stabbed cells Live and dead cells were detected using calcein green AM (Molecular Probes) and propidium iodide (Molecular Probes), respectively. Five minutes after stabbing, cells were exposed to a mixture of the two dyes. After incubation for $30 \mathrm{~min}$, green (live) and dead (red) cells were counted.

\section{Acknowledgments}

This work was partly supported by grants from a Japan MEXT Grant-in-Aid for Scientific Research on priority areas, NEDO (the New Energy and Industrial Technology Development Organization), HFSP (the Human Frontier Science Program), and the Japan Society for the Promotion of Science for Young Scientists. The authors thank Drs. Chitoshi Itakura and Yuichi Watanabe at BSI Olympus Collaboration Center for technical support.

\section{Open Access}

This article is distributed under the terms of the Creative Commons Attribution Noncommercial License which permits any noncommercial use, distribution, and reproduction in any medium, provided the original author(s) and source are credited.

\section{References}

Chen, X., Kis, A., Zettle, A., and Bertozzi, R. C. (2007). A cell nanoinjector based on carbon nanotubes. Proc. Natl. Acad. Sci. USA 104, 8218-8222.

Cuerrier, M. C., Lebel, R., and Grandbois, M. (2007). Single cell transfection using plasmid decorated AFM probes. Biochem. Biophys. Res. Commun. 355, 632-636.

Felgner, L. F., Gadek, R. T., Holm, M., Roman, R., Chan, W. H., Wenz, M., Northrop, P. J., Ringold, M. G., and Danielsen, M. (1987). Lipofection: a highly efficient, lipid-mediated DNA-transfection procedure. Proc. Natl. Acad. Sci. USA 84, 7413-7417.

Graessmann, A., Graessmann, H., Hoffmann, H., and Niebel, J. (1974). Inhibition by interferon of SV40 cDNA transcribed in vitro. FEBS Lett. 39, 249-251.

Hama, H., Hara, C., Yamaguchi, K., and Miyawaki, A. (2004). PKC signaling mediates global enhancement of excitatory synaptogenesis in neurons triggered by local contact with astrocytes. Neuron 41, 405-415.

Han, S., Nakamura, C., Obataya, I., Nakamura, N., and Miyake, J. (2005). Gene expression using an ultrathin needle enabling accurate displacement and low invasiveness. Biochem. Biophys. Res. Commun. 332, 633-639.

Hass, K., Sin, W.-C., Javaherian, A., Li, Z., and Cline, H. T. (2001). Single-cell electroporation for gene transfer in vivo. Neuron 29, 583-591.

Mizuno, H., Sawano, A., Hama, H., and Miyawaki, A. (2001). Red fluorescent protein from dissociation as a fusion tag and a partner for fluorescence resonance energy transfer. Biochemistry 40, 2502-2510.

Nagai, T., Ibata, K., Park, E. S., Kubota, M., Mikoshiba, K., and Miyawaki, A. (2002). A variant of yellow fluorescent protein with fast and efficient maturation for cell-biological applications. Nat. Biotechnol. 20, 87-90.

Obataya, I., Nakamura, C., Han, S., Nakamura, N., and Miyake, J. (2005). Nanoscale operation of a living cell using an atmic force microscope with a nanoneedle. Nano Lett. 5, 27-30.

Olofsson, J., Nolkrantz, K., Ryttsen, F., Lambie, B. A., Weber, S. G., and Orwar, O. (2003). Singlecell electroporation. Curr. Opin. Biotechnol. 14, 29-34. 
Hara et al.

Stephens, J. D. and Pepperkok, R. (2001). The many ways to cross the plasma membrane. Proc. Natl. Acad. Sci. USA 98, 4295-4298.

Stevens, B. (2003). Glia: much more than the neuron's side-kick. Curr. Biol. 13, 469-472.

Stevenson, D., Agate, B., Tsampoula, X., Fischer, P., Brown, T. A. C., Sibbett, A. R., GunnMoore, F., and Dholokia, K. (2006). Femtosec-
A practical device for pinpoint delivery of molecules

ond optical transfection of cells: viability and efficiency. Opt. Express 16, 7125-7133.

Tirlapur, U. K. and Konig, K. (2002). Target transfection by femtosecond laser. Nature 290, 290-291.

Tseng, A. A. (2005). Recent developments in nanofabrication using focused ion beams. Small 1, 924-939. 